\title{
Um Algoritmo de Decodificação Iterativa $q$-ária para Códigos Turbo Produto
}

\author{
Daniel C. Cunha ${ }^{1}$, Vagner V. do Nascimento ${ }^{2}$ e Jaime Portugheis ${ }^{3}$
}

\begin{abstract}
Resumo-Este artigo considera o estudo de esquemas de codificação turbo produto construídos a partir de códigos Reed-Solomon componentes. Uma proposta de modificação do algoritmo de Chase é desenvolvida para satisfazer requisitos dos símbolos $q$-ários e utilizada na implementação de um algoritmo de decodificação iterativa $q$-ária. É verificado que o algoritmo de decodificação iterativa $q$-ária apresenta uma redução da complexidade quando comparado ao algoritmo turbo binário. Resultados de simulação são apresentados para mostrar a eficácia do algoritmo proposto.
\end{abstract}

Palavras-Chave-Decodificação de Chase, Códigos produto, Confiabilidade $q$-ária.

Abstract-This article considers the study of turbo product encoding schemes constructed from components Reed-Solomon codes. A proposal of modification of the Chase algorithm is developed to satisfy $q$-ary symbols requirements and is used in the implementation of a iterative $q$-ary decoding algorithm. It is verified that the iterative $q$-ary decoding algorithm presents a reduction of complexity when compared to the binary turbo algorithm. Simulation results are presented to show the effectiveness of the proposed algorithm.

Keywords - Chase decoding, Product codes, $q$-ary reliability.

\section{INTRODUÇÃO}

Desde a sua introdução em [1], a decodificação iterativa (turbo) de códigos produto ganhou muita importância no desenvolvimento de sistemas de transmissão digital com códigos corretores de erros. Ela já é empregada em sistemas de transmissão por satélite e se tornou uma opção tanto em redes metropolitanas sem fio como também em enlaces ópticos de alta velocidade [2]. Códigos produto são na sua forma padrão construídos através da concatenação serial de dois códigos de bloco lineares binários ou $q$-ários, separados por um entrelaçador linha-coluna a nível de símbolo. Os códigos RS têm sido empregados como códigos $q$-ários componentes da concatenação serial. Em [3] e [4] foram analisados códigos RS corretores de erros múltiplos e em [2], códigos RS corretores de erros simples. Entretanto, em todos os trabalhos citados anteriormente, cada símbolo $q$-ário é representado por bits e uma matriz de confiabilidades dos bits observados é montada no receptor. Sendo assim, o algoritmo de Chase binário pode ser aplicado na decodificação das linhas e colunas do código produto.

1 Departamento de Engenharia Elétrica, POLI, Universidade de Pernambuco (UPE), Recife-PE, Brasil.

2 Gerência de Tecnologia da Informação e Telecomunicações, Petróleo Brasileiro S.A. (PETROBRAS), Aracaju-SE, Brasil.

3 Departamento de Comunicações, FEEC, Universidade Estadual de Campinas (UNICAMP), Campinas-SP, Brasil.

E-mails: dccunha@upe.poli.br,_ vagnervale@gmail.com jaime@decom.fee.unicamp.br.
Este trabalho considera sistemas de transmissão digital com códigos produto construídos a partir de códigos RS como códigos componentes. No receptor, uma matriz de confiabilidades dos símbolos $q$-ários observados é montada. Como consequência disto, um proposta de adaptação do algoritmo de Chase para satisfazer requisitos dos símbolos $q$-ários foi desenvolvida. Esta adaptação implica em uma redução da complexidade de decodificação quando comparada com a decodificação turbo binária. A adaptação sugerida permite uma realimentação apropriada dos símbolos $q$-ários e suas confiabilidades a cada iteração do decodificador turbo.

$\mathrm{O}$ artigo está organizado como se segue. $\mathrm{O}$ algoritmo Chase $q$-ário é descrito na seção II. Na seção III, o algoritmo turbo $q$-ário é detalhado. Na seção IV, os algoritmos turbo $q$-ário e o turbo binário são comparados através do compromisso entre complexidade e desempenho dos mesmos. Por fim, comentários finais são realizados na seção V.

\section{ALGORITMO DE CHASE $q$-ÁRIO}

Considere um código de bloco linear $C(n, k, d)$, em que $n$ é o tamanho das palavras-código, $k$ é o número de símbolos de informação e $d$ é a distância de Hamming mínima do código. Com o objetivo de melhorar o desempenho da decodificação de códigos de bloco realizada por decisão abrupta, D. Chase propôs em [5] uma classe de algoritmos de decodificação que utiliza informação de medida de canal. Estes algoritmos são descritos a seguir.

Considere a sequência $\mathbf{Y}=\left(y_{1}, \ldots, y_{l}, \ldots, y_{n}\right)$, em que $y_{l} \in$ $\mathrm{GF}(q), q=2^{m}, m=1$, obtida por decisão abrupta da palavra recebida $\mathbf{R}=\left(r_{1}, \ldots, r_{l}, \ldots, r_{n}\right)$. Para altos valores de Relação Sinal-Ruído (RSR), a palavra-código correta $\mathbf{C}=$ $\left(c_{1}, \ldots, c_{l}, \ldots, c_{n}\right)$ é localizada, com probabilidade alta, em uma esfera de raio $(d-1)$ centrada no vetor $\mathbf{Y}$. A partir daí, a sequência $\mathbf{Y}$ pode ser perturbada por um padrão de teste $\mathbf{T}$ para se gerar uma nova sequência

$$
\mathbf{Y}^{\prime}=\mathbf{Y} \oplus \mathbf{T},
$$

em que $\oplus$ representa a operação soma módulo 2. A alteração da sequência $\mathbf{Y}$ por $\mathbf{T}$ tem por objetivo encontrar a palavra-código correta $\mathbf{C}$ dentro da esfera de raio $(d-1)$. Usando esta regra, o desempenho da decodificação pode se aproximar do desempenho do processo de decodificação completa sem a complexidade de procura da palavra-código correta em uma esfera de raio $(d-1)$. Este método de decodificação é possível de ser executado devido à informação de medida de canal, ou confiabilidade, extraída a partir da palavra observada na saída do canal. Os métodos completos de decodificação de Chase são descritos em [5]. 
Os algoritmos de Chase propostos em [5] foram aplicados apenas para códigos binários. A utilização da decodificação de Chase para códigos $q$-ários necessita de conversões bit-símbolo e símbolo-bit que podem afetar a precisão do cálculo de confiabilidade do símbolo, principalmente em sistemas que utilizam um esquema de modulação com mais de dois sinais. Com isso, as conversões mencionadas podem comprometer o desempenho da decodificação de Chase.

Para ilustrar esta perda de desempenho, considere uma aplicação que utiliza o código RS $(7,3,5)$, com capacidade de correção $t_{c}=\left\lfloor\frac{d-1}{2}\right\rfloor=2$. Suponha que a palavra-código $\mathbf{X}_{A}$ seja transmitida e que a palavra recebida na entrada do decodificador seja $\mathbf{Y}$, de acordo com a Fig. 1.

Palavra-código transmitida:

$$
\begin{aligned}
& \mathrm{X}_{\mathrm{A}}=\left[\begin{array}{lllllll}
3 & 1 & 7 & 7 & 5 & 1 & 5
\end{array}\right]
\end{aligned}
$$

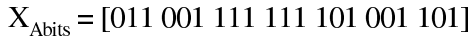

Palavra recebida:

$$
\begin{aligned}
& \mathrm{Y}=\left[\begin{array}{lllllll}
4 & 1 & 7 & 0 & 5 & 6 & 2
\end{array}\right] \\
& \mathrm{Y}_{\text {bits }}=\left[\begin{array}{llll}
100001 & 111000101110010
\end{array}\right]
\end{aligned}
$$

Erros:

$$
\begin{aligned}
& \mathrm{E}=\mathrm{Y}-\mathrm{X}_{\mathrm{A}}=\left[\begin{array}{lllllll}
1 & 0 & 0 & 1 & 0 & 5 & 5
\end{array}\right]
\end{aligned}
$$

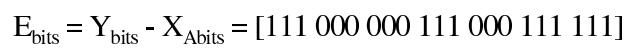

Fig. 1. Exemplo de erro em uma palavra do código $\mathrm{RS}(7,3,5)$ transmitida.

Fazendo-se as conversões símbolo-bit das sequências $\mathbf{X}_{A}$ e $\mathbf{Y}$, são geradas as sequências $\mathbf{X}_{\text {Abits }} \mathrm{e} \mathbf{Y}_{\text {bits }}$, respectivamente. Considerando a utilização do Algoritmo de Chase II para aumentar a capacidade de correção do código, é possível adicionar $2^{\lfloor d / 2\rfloor}=2^{\lfloor 5 / 2\rfloor}=4$ padrões de teste $\mathbf{T}$ à palavra $\mathbf{Y}_{\text {bits }}$, permitindo, no máximo, a modificação das $p=$ $\lfloor d / 2\rfloor$ posições de menores confiabilidades da palavra recebida $\mathbf{Y}_{\text {bits }}$. Entretanto, mesmo com a aplicação do Algoritmo de Chase II à sequência $\mathbf{Y}_{\text {bits }}$ e uma posterior conversão bit-símbolo para se obter a nova sequência $\mathbf{Y}^{\prime}$, o decodificador algébrico não conseguiria decodificar $\mathbf{Y}^{\prime}$ corretamente, pois ele é capaz de corrigir no máximo $t_{c}=2$ erros de símbolos. Para decodificar a palavra recebida $\mathbf{Y}$ de maneira correta, por meio da técnica de Chase, seria necessário manipular a palavra $\mathbf{Y}_{\text {bits }}$ em pelo menos 2 grupos de 3 posições (bits) consecutivas, totalizando 6 posições. É possível desenvolver um algoritmo que atenda esta necessidade, porém ele teria uma quantidade grande de padrões de teste e, consequentemente, seria mais complexo.

Com o intuito de melhorar o desempenho da decodificação para códigos $q$-ários, é proposta uma modificação no método de Chase. Ela é baseada no Algoritmo de Chase II devido à sua boa relação entre desempenho e complexidade. Considere novamente o exemplo do código $\operatorname{RS}(7,3,5)$ e assuma que a palavra recebida é a sequência $\mathbf{Y}$ em substituição à sequência $\mathbf{Y}_{\text {bits }}$, oriunda da conversão símbolo-bit. Assuma que são gerados padrões de teste que permitam a substituição dos símbolos $q$-ários das posições com baixa confiabilidade por outros símbolos $q$-ários mais adequados. Desta maneira, são necessárias substituições em apenas duas posições de $\mathbf{Y}$ para que o decodificador algébrico consiga decodificar a nova sequência $\mathbf{Y}^{\prime}$ em uma palavra-código válida do código

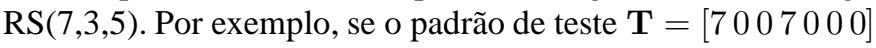
for somado (módulo 8) à palavra recebida $\mathbf{Y}$ indicada na Fig. 1, será obtida a nova palavra $\mathbf{Y}^{\prime}$, dada por

$$
\begin{aligned}
\mathbf{Y}^{\prime} & =\mathbf{Y}+\mathbf{T} \\
& =\left[\begin{array}{lllllll}
4 & 1 & 7 & 0 & 5 & 6 & 2
\end{array}\right]+\left[\begin{array}{lllllll}
7 & 0 & 0 & 7 & 0 & 0 & 0
\end{array}\right] \\
& =\left[\begin{array}{lllllll}
3 & 1 & 7 & 7 & 5 & 6 & 2
\end{array}\right] .
\end{aligned}
$$

Note que a sequência $\mathbf{Y}^{\prime}$ agora pode ser decodificada pelo decodificador algébrico $q$-ário, pois possui apenas dois erros de símbolo quando comparada à sequência $\mathbf{X}_{A}$.

Segundo o exemplo anterior, o método de Chase modificado para suportar a decodificação de códigos $q$-ários, sem a necessidade de conversões símbolo-bit e bit-símbolo, deve seguir o mesmo algoritmo definido em [5]. Todavia, a obtenção do conjunto de padrões de teste difere das formas empregadas nas três versões dos algoritmos originais de Chase [5], pois agora são considerados símbolos $q$-ários. Logo, a primeira mudança significativa para a proposta do algoritmo de decodificação $q$-ário, é a modificação da geração de padrões de teste.

O Algoritmo de Chase II define o conjunto de padrões de teste como todas as possíveis combinações de 1's nas $\lfloor d / 2\rfloor$ posições de menor confiabilidade da palavra recebida e zeros nas demais posições. O número de padrões de teste poderia ser alterado simplesmente aumentando-se a quantidade de posições não confiáveis da palavra $\mathbf{Y}_{\text {bits }}$ proporcionalmente ao aumento do número de posições da sequência $\mathbf{Y}$ para a sequência $\mathbf{Y}_{\text {bits }}$, ou seja, $\log _{2}(q)$ vezes. Desta maneira, o número de posições não confiáveis de $\mathbf{Y}_{\text {bits }}$ passaria a ser $\lfloor d / 2\rfloor \log _{2}(q)$, resultando em uma quantidade de padrões de teste dada pela expressão:

$$
2^{\lfloor d / 2\rfloor \cdot \log _{2}(q)}=\left(2^{\log _{2}(q)}\right)^{\lfloor d / 2\rfloor}=q^{\lfloor d / 2\rfloor} .
$$

Apesar desta ser uma solução simples, a expressão (2) indica que a quantidade de padrões de teste tende a crescer exponencialmente com a dimensão do corpo de Galois do código $q$-ário utilizado. Logo, a aplicação deste algoritmo de Chase modificado seria impraticável devido à sua alta complexidade. Para reforçar o que foi dito, considere novamente o código $\operatorname{RS}(7,3,5)$ sobre o $\mathrm{GF}(8)$. A alteração do número de padrões de teste sugerida pela expressão (2) geraria um aumento de $2^{\lfloor d / 2\rfloor}=2^{\lfloor 5 / 2\rfloor}=4$ para $q^{\lfloor d / 2\rfloor}=8^{\lfloor 5 / 2\rfloor}=$ 64 padrões de teste.

Para reduzir a complexidade da modificação do algoritmo de Chase proposta, é necessário uma alteração no cálculo da confiabilidade do símbolo recebido. Sabe-se que a sequência Y é gerada no demodulador por meio de decisão abrupta e que o símbolo escolhido $y_{l}=\gamma_{0}$ obedece à seguinte expressão:

$$
P\left(y_{l}=\gamma_{0} \mid r_{l}\right)>P\left(y_{l}=\gamma \mid r_{l}\right), \quad \forall \gamma \neq \gamma_{0},
$$

em que $r_{l}$ é uma estatística suficiente para o $l$-ésimo símbolo transmitido e $\gamma_{0}, \gamma \in G F(q)$.

Fazendo uso de uma inequação similar à (3), é possível gerar uma segunda sequência $\mathbf{Y}^{a}$, obtida também por decisão 
abrupta, com os símbolos $y_{l}^{a}=\gamma_{1}$ tal que:

$$
\begin{aligned}
& P\left(y_{l}=\gamma_{0} \mid r_{l}\right)>P\left(y_{l}=\gamma_{1} \mid r_{l}\right)>P\left(y_{l}=\gamma \mid r_{l}\right), \\
& \forall \gamma \neq \gamma_{1} \neq \gamma_{0},
\end{aligned}
$$

ou seja, a sequência $\mathbf{Y}^{a}$ conterá os símbolos $y_{l}^{a}$ com a segunda maior probabilidade de terem sido transmitidos dado que $r_{l}$ foi observado.

Utilizando $\mathbf{Y}^{a}$, a sequência $\mathbf{Y}^{\prime}$ pode assumir, alternativamente, os símbolos provenientes das $\lfloor d / 2\rfloor$ posições menos confiáveis das sequências $\mathbf{Y}$ e $\mathbf{Y}^{a}$. Sendo assim, os símbolos do padrão de teste são obtidos como

$$
t_{l}=\left\{\begin{array}{cc}
y_{l}^{a}-y_{l}, & y_{l}^{\prime}=y_{l}^{a} \\
0, & y_{l}^{\prime}=y_{l}
\end{array} .\right.
$$

Com isto, teremos um conjunto $q$-ário com apenas $2^{\lfloor d / 2\rfloor}$ padrões de teste, ou seja, a mesma quantidade do método binário.

Semelhante ao caso binário, a confiabilidade de um símbolo $q$-ário recebido $y_{l}$ pode ser calculada por meio de Razão de Log-Verossimilhança (LLR, do inglês Log-Likelihood Ratio). Entretanto, para o caso $q$-ário, são considerados todos os símbolos do alfabeto $G F(q)$ para gerar a seguinte LLR:

$$
\Lambda\left(y_{l} \mid r_{l}\right)=\ln \left(\frac{P\left(y_{l}^{(1)} \mid r_{l}\right)}{\sum_{b=2}^{q} P\left(y_{l}^{(b)} \mid r_{l}\right)}\right),
$$

em que $y_{l}^{(b)}$ é o símbolo $q$-ário com a $b$-ésima maior probabilidade a posteriori.

Note que o símbolo $y_{l}^{(1)}$ representa a $l$-ésima posição obtida a partir da sequência $\mathbf{Y}$ e o símbolo $y_{l}^{(2)}$ é a $l$-ésima posição obtida a partir da sequência $\mathbf{Y}^{a}$. Aplicando a regra de Bayes e considerando $P\left(y_{l}^{(b)}\right)$ constante, a equação (6) pode ser escrita como

$$
\Lambda\left(y_{l} \mid r_{l}\right)=\ln \left(\frac{P\left(r_{l} \mid y_{l}^{(1)}\right)}{\sum_{b=2}^{q} P\left(r_{l} \mid y_{l}^{(b)}\right)}\right) .
$$

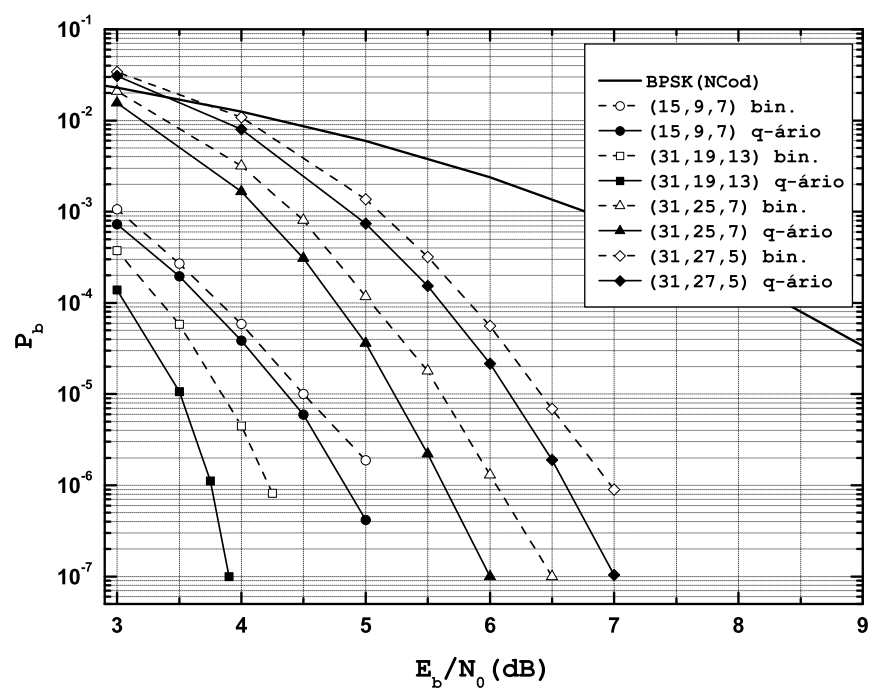

Fig. 2. Comparação de desempenho das decodificações de Chase binária e $q$-ária para códigos RS típicos.

A Fig. 2 ilustra o desempenho dos algoritmos de decodificação de Chase binário (Chase II) e $q$-ário para os códigos Reed-Solomon (RS) $(15,9,7),(31,19,13),(31,25,7)$ e $(31,27,5)$ por meio da curva de taxa de erro de bit $\left(P_{b}\right)$ versus $E_{b} / N_{0}$, em que $E_{b}$ é a energia média por bit de informação e $N_{0}$, a densidade espectral de potência do ruído AWGN. A modulação BPSK (do inglês, Binary Phase Shift Keying) é considerada. Para o código RS $(15,9,7)$ e considerando que $E_{b} / N_{0}=5 \mathrm{~dB}$, verifica-se uma redução de 5 vezes na taxa de erro de bit em favor do algoritmo proposto. Em relação aos códigos RS $(31,25,7)$ e $(31,27,5)$, tem-se uma redução da $P_{b}$ de aproximadamente 10 vezes para $E_{b} / N_{0}=6$ e $7 \mathrm{~dB}$, respectivamente. Por fim, o código RS $(31,19,13)$ apresenta uma redução de 20 vezes em $P_{b}$ para $E_{b} / N_{0}=3,75 \mathrm{~dB}$ ao se utilizar o algoritmo de decodificação $q$-ário.

\section{DECODIFICAÇÃO TURBO $q$-ÁRIA}

\section{A. Códigos Produto}

Códigos produto foram introduzidos por Elias [6] e representam uma maneira simples e relativamente eficiente para a construção de códigos de bloco longos a partir de códigos componentes curtos. Códigos produto bidimensionais clássicos são obtidos a partir da concatenação serial de dois códigos de bloco lineares $C_{1}\left(n_{1}, k_{1}, d_{1}\right)$ e $C_{2}\left(n_{2}, k_{2}, d_{2}\right)$ sobre $\mathrm{GF}(q)$. O código produto resultante $P\left(n_{p}, k_{p}, d_{p}\right)$ possui comprimento $n_{p}=n_{1} n_{2}, k_{p}=k_{1} k_{2}$ símbolos de informação e distância mínima $d_{p}=d_{1} d_{2}$. As $n_{1}$ linhas do código produto são palavras-código de $C_{1}$ e as $n_{2}$ colunas são palavras-código de $C_{2}$ [7]. Neste trabalho, consideraremos a utilização de códigos produto Reed-Solomon (RS) compostos por códigos componentes RS sobre GF $(q)$ idênticos.

\section{B. Decodificação Turbo q-ária de Códigos Produto}

As linhas e colunas de um código produto $P$ podem ser decodificadas por um método iterativo chamado decodificação turbo [1]. Seja $\boldsymbol{\Lambda}(\mathbf{Y})$ a matriz de confiabilidade dos símbolos recebidos. O diagrama do decodificador turbo binário é ilustrado na Fig. 3 (conforme as linhas cheias).

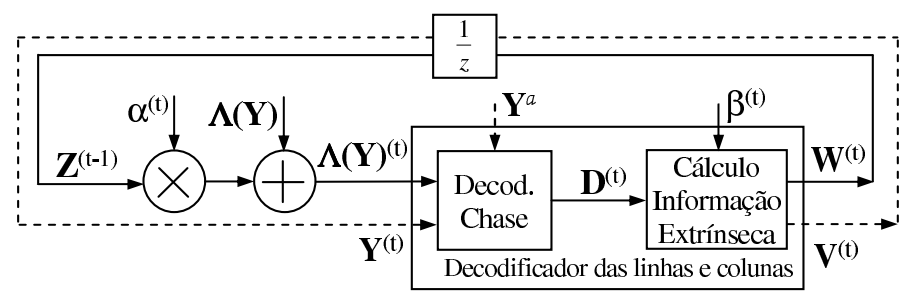

Fig. 3. Diagrama do decodificador turbo produto.

Primeiramente, o decodificador executa a decodificação suave (decodificação de Chase) das linhas (ou colunas) de $P$ utilizando $\boldsymbol{\Lambda}(\mathbf{Y})$ como a matriz de entrada. Assim, a matriz decodificada por Chase, $\mathbf{D}^{(t=1)}$, é gerada depois da primeira iteração $(t=1)$. A entrada suave para a $t$-ésima iteração do decodificador é dada por

$$
\Lambda(\mathbf{Y})^{(t)}=\Lambda(\mathbf{Y})+\alpha^{(t)} \mathbf{Z}^{(t-1)},
$$


em que $\alpha^{(t)}$ é um fator de escala (vide [8] e [9]) que varia no intervalo $[0,0 ; 1,0]$ de acordo com [1].

De acordo com [10], a saída suave $\boldsymbol{\Lambda}\left(d_{i j}\right)^{(t)}$ pode ser aproximada por

$$
\boldsymbol{\Lambda}\left(d_{i j}\right)^{(t)}=\beta^{(t)} \mathbf{\Phi}\left(d_{i j}\right)^{(t)},
$$

em que $\beta^{(t)}$ é um fator de confiabilidade que varia no intervalo $[0,2 ; 1,0]$ segundo [1] e $\mathbf{\Phi}\left(d_{i j}\right)^{(t)}$ é a LLR da decisão $d_{i j}^{(t)}$, que é definida por:

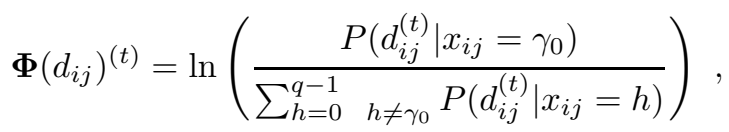

em que $\mathbf{X}$ é a matriz obtida na saída do codificador produto e $\gamma_{0} \in G F(q)$.

Desta maneira, a informação extrínseca é dada por:

$$
\mathbf{W}^{(t)}=\boldsymbol{\Lambda}\left(d_{i j}\right)^{(t)}-\boldsymbol{\Lambda}(\mathbf{Y})^{(t)} .
$$

$\mathrm{O}$ algoritmo utiliza a matriz de sequências $\mathbf{Y}^{a}$ e a matriz $\boldsymbol{\Lambda}(\mathbf{Y})$ que são obtidas de acordo com as equações (4) e (7), respectivamente. No caso binário, podemos identificar o bit $y_{i j}$ pelo sinal da sua confiabilidade $\boldsymbol{\Lambda}\left(y_{i j}\right)$ (positivo ou negativo). Entretanto, no caso $q$-ário, o valor do símbolo não está implícito na confiabilidade, porque o símbolo pode assumir $q$ valores de acordo com o alfabeto utilizado. Assim, o decodificador turbo $q$-ário deve ser adicionalmente alimentado com as matrizes de sequências $\mathbf{Y}^{(0)}=\mathbf{Y}$ e $\mathbf{Y}^{a}$.

Em cada iteração do decodificador turbo $q$-ário, o valor do símbolo $y_{i j}^{(t)}$ da $t$-ésima matriz de entrada $\mathbf{Y}^{(t)}$ deve ser comparado com o valor do símbolo $d_{i j}^{(t)}$ da $t$-ésima matriz decodificada $\mathbf{D}^{(t)}$. Se $y_{i j}^{(t)}=d_{i j}^{(t)}$, significa que o decodificador de Chase $q$-ário não corrigiu o símbolo de entrada. Desta maneira, a informação extrínseca $\mathbf{W}^{(t)}$ para a próxima iteração deve ser obtida conforme é ilustrado na Fig. 3 (linhas tracejadas). Observa-se ainda que a $t$-ésima matriz de símbolos decodificados $\mathbf{V}^{(t)}$ deve retornar à entrada do decodificador $q$-ário. Sendo assim, quando $y_{i j}^{(t)} \neq d_{i j}^{(t)}$, um método diferente para a realimentação do decodificador turbo $q$-ário é proposto conforme ilustrado na Fig. 4 e descrito a seguir.

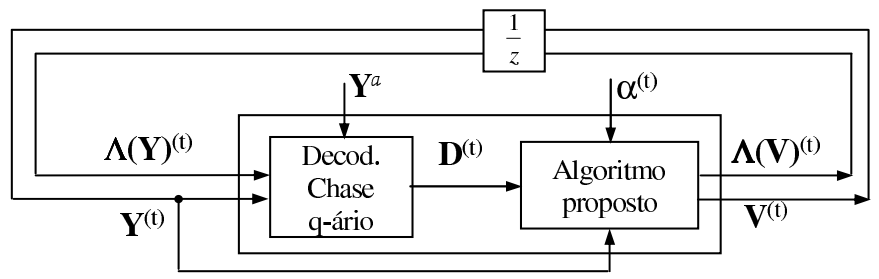

Fig. 4. Decodificador turbo $q$-ário para $y_{i j}^{(t)} \neq d_{i j}^{(t)}$.

No caso binário, o bit é identificado através da confiabilidade. Desta maneira, pode-se considerar que o valor da confiabilidade $\boldsymbol{\Lambda}\left(y_{i j}\right)$ varia dentro de um segmento de reta de intervalo $[-1 ;+1]$, de acordo com a Fig. 5a. Devido à diversidade de símbolos, não é possível fazer esta mesma consideração no caso $q$-ário. Logo, quando $y_{i j}^{(t)} \neq d_{i j}^{(t)}$, o método proposto, ilustrado na Fig. 5b, é aplicado para calcular o valor da confiabilidade para próxima iteração, agora denotada por $\Lambda\left(v_{i j}\right)^{(t)}$. Na Fig. 5b, para a $t$-ésima iteração, o ponto $A$ no eixo das abscissas representa o valor da confiabilidade $\Lambda\left(y_{i j}\right)^{(t)}$ do símbolo de entrada, enquanto o ponto $B$ no eixo das ordenadas representa o valor da confiabilidade $\Lambda\left(d_{i j}\right)^{(t)}$ do símbolo decodificado pelo algoritmo de Chase $q$-ário. Podemos encontrar o ponto $C$ traçando uma reta perpendicular ao segmento $\overline{A B}$ de tal forma que o segmento $\overline{A C}$ obedeça à expressão $\overline{A C}=\alpha^{(t)} \times \overline{A B}$. Por meio de alguns cálculos trigonométricos básicos, podemos obter o segmento $\overline{O D}$, cujo valor representará a confiabilidade $\Lambda\left(v_{i j}\right)^{(t)}$ da próxima iteração. Note que, dependendo de $A$, $B$ e $\alpha^{(t)}$, o ponto $D$ poderá estar nos eixos das abscissas $\Lambda\left(y_{i j}\right)^{(t)}$ ou das ordenadas $\Lambda\left(d_{i j}\right)^{(t)}$ e sua posição definirá se o símbolo de entrada da próxima iteração $v_{i j}$ será igual a $y_{i j}$ ou $d_{i j}$, respectivamente.

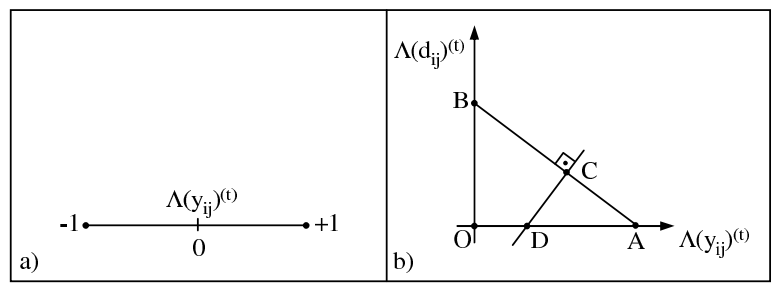

Fig. 5. Projeção da decodificação turbo: a) binária e b) $q$-ária.

\section{COMPARAÇÃO DE COMPLEXIDADE VERSUS DESEMPENHO}

Na seção II, uma modificação do algoritmo de Chase foi proposta para códigos $q$-ários. Este algoritmo foi empregado em uma nova proposta de algoritmo de decodificação turbo apresentada na seção III. A partir deste momento, faremos uma comparação de complexidade e desempenho do algoritmo turbo $q$-ário proposto e o algoritmo turbo binário definido por Pyndiah em [1].

Como referência para a comparação, foram considerados os resultados obtidos em [4] para o algoritmo turbo binário ([1]). Foram utilizados os fatores $\alpha^{(s)}$ e $\beta^{(s)}$ variando a cada meia iteração $s$ (linha/coluna) do decodificador e assumindo os valores estabelecidos em [1]. Os códigos produto considerados foram compostos pelos códigos RS $(31,27,5)$ e $(63,57,7)$. $\mathrm{O}$ algoritmo de Berlekamp-Massey (BM) foi utilizado para a decodificação de decisão abrupta e o parâmetro $p$ do algoritmo de Chase foi considerado igual a 4 . Por fim, $t=4$ iterações completas do decodificador turbo foram realizadas.

Para o algoritmo turbo $q$-ário proposto neste trabalho, foram usados os mesmos códigos RS componentes mencionados anteriormente, assim como o algoritmo de BM. Em relação aos fatores $\alpha$ e $\beta$, foram considerados os valores $\alpha^{(s)}=[0,0 ; 0,2 ; 0,3 ; 0,5 ; 0,7 ; 0,9 ; 1,0 ; 1,0] \mathrm{e}$ $\beta^{(s)}=[0,2 ; 0,4 ; 0,6 ; 0,8 ; 1,0 ; 1,0 ; 1,0 ; 1,0]$. Finalmente, o parâmetro $p$ do algoritmo de Chase $q$-ário foi estabelecido de acordo com o algoritmo de Chase original, ou seja, $p=\lfloor d / 2\rfloor$.

Sejam $N_{s}, N_{m}, N_{c}$ e $N_{g}$, as operações de soma, multiplicação, comparação e soma módulo 2 , respectivamente. Estas operações foram selecionadas para avaliar a 
complexidade computacional de cada algoritmo, baseado no que foi empregado em [11]. Na Tabela I, são indicadas as operações referentes ao algoritmo turbo binário. É importante ressaltar que a análise realizada em [11] levou em consideração o uso de códigos de Hamming estendidos. Para o nosso caso, em que códigos RS são considerados, alguns passos do algoritmo foram adaptados.

Com o intuito de computar a quantidade de operações do algoritmo turbo $q$-ário, este será descrito conforme os seguintes passos:

1) Encontrar os dois símbolos com as duas maiores probabilidades a posteriori $P\left(y_{l}^{(1)} \mid r_{l}\right)$ e $P\left(y_{l}^{(2)} \mid r_{l}\right)$, a partir das probabilidades a posteriori dos bits recebidos, e gerar os símbolos referentes às seqüências $\mathbf{Y}$ e $\mathbf{Y}^{a}$, respectivamente.

2) Calcular a confiabilidade de cada símbolo da seqüência $\mathrm{Y}$

$$
\Lambda\left(y_{l} \mid r_{l}\right)=\ln \left(\frac{P\left(y_{l}^{(1)} \mid r_{l}\right)}{1-P\left(y_{l}^{(1)} \mid r_{l}\right)}\right), l=1, \ldots, n .
$$

3) Determinar as $p$ posições menos confiáveis da seqüência Y.

4) Gerar os $2^{p}$ padrões de teste e suas respectivas seqüências perturbadas $\mathbf{Y}^{\prime}$. Para isso, é necessário apenas substituir os símbolos das posições menos confiáveis de $\mathbf{Y}$ pelos símbolos referentes em $\mathbf{Y}^{a}$ de acordo com os padrões de teste.

5) Decodificar a sequência perturbada $\mathbf{Y}^{\prime}$ por meio do algoritmo de BM.

6) Calcular o peso analógico

$$
W_{\Lambda}\left(\mathbf{Z}^{e}\right)=\sum_{i=1}^{n} \Lambda_{i} \cdot z_{i}^{e}
$$

em que $\mathbf{Z}^{e}$ é o padrão de erro q-ário $\mathbf{E}$ com as posições não nulas substituídas por 1 .

7) Obter a palavra decodificada $\mathbf{D}$ por Chase a partir do padrão de erro de menor peso analógico $\left(\mathbf{E}_{\min }\right)$.

8) Calcular a confiabilidade da decodificação, considerando o canal AWGN. Observe que, conforme [10], calculamos as confiabilidades para os bits

$$
\Lambda\left(d_{i j, f}\right)=\beta \sqrt{E_{b}},
$$

em que $f$ varia de 0 a $m-1$. Em seguida, geramos as confiabilidades para os símbolos.

9) Calcular os valores das confiabilidades e dos símbolos para próxima iteração segundo o procedimento proposto na seção III.

Na Tabela II, são indicadas as operações contabilizadas para o algoritmo turbo $q$-ário. Vale ressaltar que as operações referentes aos dois algoritmos consideram apenas a complexidade computacional de uma palavra-código componente. Além disso, os passos 4 (Tabela I) e 5 (Tabela II) se referem à decodificação de $\mathrm{BM}$ e, por gerar o número de operações nos dois casos, não são levados em conta para a comparação de complexidade.
TABELA I

COMPLEXIDADE DE DECODIFICAÇÃO DO ALGORITMO TURBO BINÁRIO.

\begin{tabular}{c||c||c||c||c}
\hline Passo & $N_{s}$ & $N_{m}$ & $N_{c}$ & $N_{g}$ \\
\hline \hline 1 & - & - & $m n(p+1)$ & - \\
\hline 2 & - & - & - & $p 2^{p}$ \\
\hline 3 & - & - & - & $m n 2^{p}$ \\
\hline 4 & - & - & - & - \\
\hline 5 & $m n 2^{p}$ & $m n 2^{p}$ & - & - \\
\hline 6 & - & - & $2^{p}$ & - \\
\hline 7 & $2 m n$ & $2 m n$ & $m n\left(2^{p}-1\right)$ & - \\
\hline \hline
\end{tabular}

Para consolidar a comparação entre as complexidades dos algoritmos turbo binário e $q$-ário, definimos a Razão de Complexidade (RC) como a razão entre os números totais de operações $\left(N_{s}+N_{m}+N_{c}+N_{g}\right)$ dos algoritmos binário e $q$-ário. Para o código $\operatorname{RS}(31,27,5)^{2}$, foi obtido um valor de $\mathrm{RC}$ aproximadamente igual a 8,6 , enquanto para o código RS $(63,57,7)^{2}$, o valor de RC aproximado foi de 7,7 , resultando em uma menor complexidade do algoritmo turbo $q$-ário.

TABELA II

COMPLEXIDADE DE DECODIFICAÇÃO DO ALGORITMO TURBO $q$-ÁRIO.

\begin{tabular}{c||c||c||c||c}
\hline Passo & $N_{s}$ & $N_{m}$ & $N_{c}$ & $N_{g}$ \\
\hline \hline 1 & - & $(m-1) n$ & $(2 m-1) n$ & - \\
\hline 2 & $n$ & $n$ & - & - \\
\hline 3 & - & - & $n p-\sum_{i=1}^{p} i$ & - \\
\hline 4 & - & - & $\left(2^{p}-1\right) p$ & - \\
\hline 5 & - & - & - & - \\
\hline 6 & $(n-1) 2^{p}$ & $n 2^{p}$ & - & - \\
\hline 7 & - & - & $2^{p}-1$ & - \\
\hline 8 & - & $(2 m-1) n$ & - & - \\
\hline 9 & $2 n$ & $4 n$ & $2 n$ & - \\
\hline \hline
\end{tabular}

Na Fig. 6 é ilustrado o desempenho dos algoritmos turbo binário e $q$-ário em um canal AWGN. As curvas referentes à decodificação turbo binária foram obtidas a partir de [4]. A modulação BPSK foi considerada. Os códigos produto $\operatorname{RS}(31,27,5)^{2}$ e $\operatorname{RS}(63,57,7)^{2}$ foram utilizados e as curvas $P_{b}$ versus $E_{b} / N_{0}$ da decodificação turbo $q$-ária foram geradas através de simulações.

Para o código produto $\operatorname{RS}(31,27,5)^{2}$, foram transmitidos $\sim 2,2 \cdot 10^{8}$ bits de informação. Observe que, para alcançar uma $P_{b}=10^{-5}$, a decodificação turbo $q$-ária necessita de uma $E_{b} / N_{0} \simeq 4,25 d B$. A decodificação turbo binária alcança esta mesma $P_{b}$ para $E_{b} / N_{0} \simeq 4,05 d B$. Apesar do algoritmo turbo binário oferecer um ganho de aproximadamente $0,2 d B$, a sua complexidade da decodificação mostra-se maior que a complexidade $q$-ária. Além disso, uma possível justificativa para o melhor desempenho do algoritmo turbo binário foi o fato de ele ter utilizado o parâmetro $p$ do algoritmo de Chase maior do que o previsto pelo algoritmo original definido em [5], ou seja, o algoritmo turbo binário utilizou um valor maior $(p=4)$ do que $\lfloor d / 2\rfloor$.

Já para o código produto $\operatorname{RS}(63,57,7)^{2}$, foram transmitidos $\sim 1,2 \cdot 10^{9}$ bits de informação. Para $E_{b} / N_{0} \simeq$ 
$4,5 d B$, percebe-se que o algoritmo turbo $q$-ário oferece, com menor complexidade computacional, uma redução de aproximadamente 5 vezes em $P_{b}$ comparado ao algoritmo turbo binário. Vale ressaltar que, assim como no caso do código $\operatorname{RS}(31,27,5)^{2}$, o algoritmo turbo binário para o código $\operatorname{RS}(63,57,7)^{2}$ utilizou o parâmetro $p=4$, diferente do algoritmo turbo $q$-ário, que utilizou $p=3$, de acordo com o algoritmo original definido em [5].

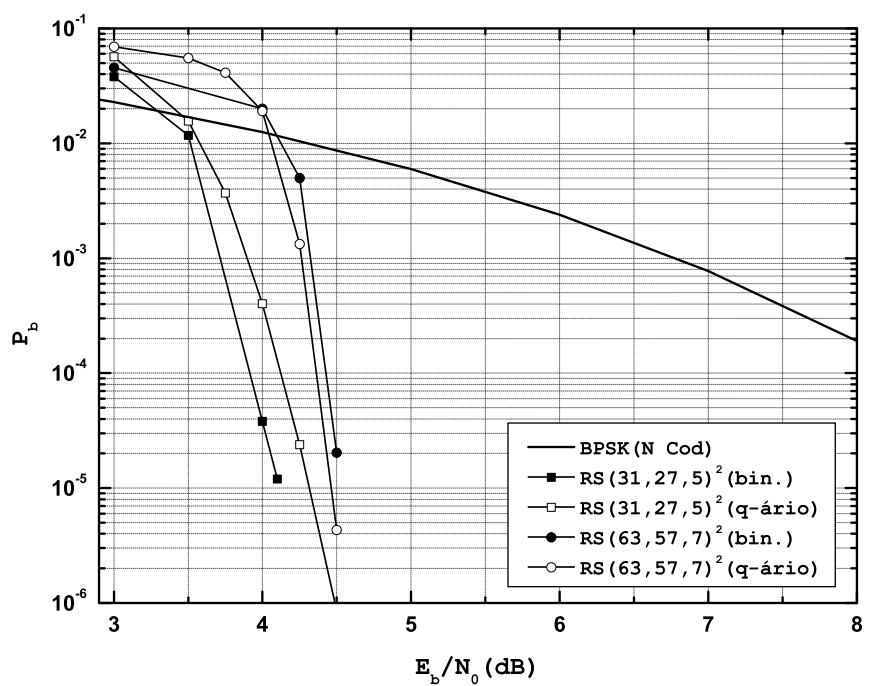

Fig. 6. Desempenho dos algoritmos de decodificação turbo binária e $q$-ária para os códigos produto RS $(31,27,5)^{2}$ e $(63,57,7)^{2}$.

\section{COMENTÁRIOS FINAIS}

Este trabalho considerou o estudo de esquemas de codificação produto construídos a partir de códigos RS componentes. Uma proposta de modificação do algoritmo de Chase binário foi desenvolvida para satisfazer requisitos dos símbolos $q$-ários. Segundo os resultados apresentados na seção II, o método de decodificação de Chase modificado para símbolos $q$-ários superou em desempenho o método original de Chase para os códigos RS apresentados, sem incrementar a complexidade de decodificação.

A modificação do algoritmo de Chase foi utilizada na implementação de um algoritmo de decodificação iterativa $q$-ária. Foi realizada uma comparação da complexidade computacional versus desempenho dos algoritmos de decodificação turbo binária e $q$-ária. A complexidade computacional foi avaliada por meio do cálculo do número de operações matemáticas efetuadas em cada um dos algoritmos. Foi observado que o algoritmo turbo $q$-ário se mostrou menos complexo que o algoritmo turbo binário para os códigos produto $\operatorname{RS}(31,27,5)^{2}$ e $\operatorname{RS}(63,57,7)^{2}$. Adicionalmente, foi verificado que o desempenho do algoritmo turbo binário foi ligeiramente superior ao algoritmo $q$-ário para o código $\operatorname{RS}(31,27,5)^{2}$, justificado pela utilização de um maior conjunto de padrões de teste no algoritmo de Chase. Por fim, ficou evidenciado o melhor desempenho do algoritmo turbo $q$-ário frente ao algoritmo binário para o código $\operatorname{RS}(63,57,7)^{2}$.

\section{AGRADECIMENTOS}

Este trabalho foi parcialmente financiado pela Fundação de Amparo à Ciência e Tecnologia do Estado de Pernambuco (FACEPE).

\section{REFERÊNCIAS}

[1] R. Pyndiah, "Near Optimum Decoding of Product Codes: Block Turbo Codes," IEEE Trans. on Communications, vol. 2, n. 8, pp. 1003-1010, Ago 1998.

[2] R. Zhou, R.L. Bidan, R. Pyndiah e A. Goalic, "Low-Complexity High-Rate Reed-Solomon Block Turbo Codes," IEEE Trans. on Communications, vol. 55, n. 9, pp. 1656-1660, Set 2007.

[3] O. Aitsab e R. Pyndiah, "Performance of Reed-Solomon Block Turbo Codes," In: Proceedings of the IEEE Global Telecommunications Conference 1996 (GLOBECOM'96), pp. 121-125, Londres, Inglaterra, Nov 1996.

[4] C. Argon, S.W. McLaughlin e T. Souvignier, "Iterative Application of the Chase Algorithm on Reed-Solomon Product Codes," In: Proceedings of the IEEE Int. Conf. on Communications 2001 (ICC'01), vol.1, pp. 320-324, Helsinque, Finlândia, Jun 2001.

[5] D. Chase, "A Class of Algorithms for Decoding Block Codes with Channel Measurement Information," IEEE Trans. on Information Theory, vol. IT-18, n. 1, pp. 170-182, Jan 1972.

[6] P. Elias, "Error-free Coding," IEEE Trans. on Information Theory, vol. IT-4, pp. 29-37, 1954.

[7] F.J. MacWilliams e N.J. Sloane, The Theory of Error Correcting Codes . Amsterdam, The Netherlands: North-Holland, 1978.

[8] C. Berrou, A. Glavieux e P. Thitimajshima, "Near Shannon Limit Error-Correcting Coding and Decoding:Turbo-Codes(1)," IEEE Int. Conf. on Communications (ICC'93), vol. 2/3, pp. 1064-1071, Mai 1993.

[9] C. Berrou e A. Glavieux, "Near Optimum Error Correcting Coding and Decoding: Turbo-Codes," IEEE Trans. on Communications, vol. 44, pp. 1261-1271, Out 1996.

[10] D. C. Cunha e J. Portugheis, "Decodificação Iterativa (Turbo) de Códigos Produto em Canais Não-Gaussianos," In: Anais do XX Simpósio Brasileiro de Telecomunicações 2003 (SBrT'03), Rio de Janeiro-RJ, Brasil, Out 2003.

[11] C. Xu, Y.-C. Liang e W.S. Leon, "A Low Complexity Decoding Algorithm for Extended Turbo Product Codes," IEEE Trans. on Wireless Communications, vol. 7, n.1, pp. 43-47, Jan 2008. 